\title{
IN PLANE CYCLIC BEHAVIOR OF MASONRY WALLS JACKETED WITH FIBER REINFORCED MORTAR AND FIBER GRIDS
}

\author{
Viorel POPA - Lecturer, PhD, Technical University of Civil Engineering, Faculty of Civil Engineering, \\ e-mail: vpopa@utcb.ro \\ Radu PASCU - Professor, PhD, Technical University of Civil Engineering, Faculty of Civil Engineering, \\ e-mail: r_pascu@utcb.ro
}

Andrei PAPURCU - PhD Student, Technical University of Civil Engineering, Faculty of Civil Engineering, e-mail: andreipapurcu@gmail.com

\begin{abstract}
Masonry buildings represent the most vulnerable part of the building stock to seismic action in Romania. The main goal of this experimental research program is to investigate the efficiency of several retrofitting solutions using fiber reinforced polymers. Research focused on the lateral strength and displacement capacity of the retrofitted specimens. The masonry walls were built using solid bricks. Glass or carbon fiber reinforced polymers (GFRP or CFRP) embedded in a fiber reinforced mortar layer were used for jacketing. Seven specimens having essentially $25 \mathrm{~cm}$ width, $1,75 \mathrm{~m}$ height and $2,10 \mathrm{~m}$ length were tested in the experimental research program. These specimens were subjected to a constant vertical compressive stress of 1,2MPa. A quasi-static load protocol was considered for the horizontal loading. This paper presents the layout of the experimental research program and some preliminary results.
\end{abstract}

Keywords: seismic, fiber reinforced mortar, glass/carbon fiber grids

\section{Introduction}

The most seismic vulnerable buildings in Romania are masonry structures. To reduce the earthquake social and economic impact, retrofitting of these structures needs to be prioritized. Adding new reinforced concrete (RC) shear walls and $\mathrm{RC}$ jacketing represents the traditional retrofitting techniques in Romania. These good technical solutions usually imply a long construction time, building operation interruption, temporary relocation of the inhabitants, alteration of the finishing or building facilities $[1,3]$.

FRP jacketing represents an alternative option for retrofitting the existing masonry buildings. The main advantages are the relatively high strength to weight ratio, short construction time and the natural corrosion resistance [2].

FRP sheets are usually bonded on structural elements using epoxy resin. Poor fire resistance, alteration of the resin behavior at high temperatures and UV sensitivity represent the main shortcomings of this solution [5]. Moreover, poor permeability to moisture vapors that might cause moisture accumulation in the walls presents a particular importance in case of historic or architectural monuments.

The use of glass or carbon fiber grids embedded in a cementitious matrix represents an alternative solution. The advantages of this solution are [5]: fair fire resistance, good compatibility with the support, good permeability to moisture vapors and easy installation by medium skilled workers. The efficiency of the retrofitting work can be further improved if fiber reinforced mortar (FRM) is used.

Experimental research on this retrofitting technique was performed within the project "Conventional and unconventional experimental methods to determine the performance level for materials, elements and structures - METEX”. Some of the obtained results are reported here. 


\section{Experimental Program}

Seven masonry specimens with different retrofitting solutions were considered in the test plan.

All specimens were $1,75 \mathrm{~m}$ in height with a rectangular cross-section of $2,10 \mathrm{~m} \times 0,25 \mathrm{~m}$ except for specimen WMET6. Specimen WMET6 had an asymmetric cross-section with a rectangular end zone of $0,25 \mathrm{~m} \times 0,25 \mathrm{~m}$ at one end and a rectangular web of $0,125 \mathrm{~m} \times 1,85 \mathrm{~m}$. Full bricks recovered from a demolished building having average compression strength of $1,45 \mathrm{MPa}$ were used. Two reinforced concrete boundary ties, located at the top and bottom end of the masonry walls, with cross-section of $0,30 \mathrm{~m} \times 0,30 \mathrm{~m}$, were used for each specimen to ensure the load transfer. These were attached to the reaction frame such as only their translation in the longitudinal and vertical direction was allowed.

Retrofitting solutions based on Carbon Fiber Reinforced Polymers (CFRP) and Glass Fiber Reinforced Polymers (GFRP) grids were implemented for all specimens. The polymeric grids were embedded in a 15-25mm think Fiber Reinforced Mortar (FRM) layer. The effectiveness of the retrofitting work considering different jacketing solutions applied on both faces of the walls or on a single face was investigated. Two reference specimens were tested: WMET1 - un-retrofitted masonry wall and WMET8 - retrofitted masonry wall just by FRM jacketing on both sides.

The characteristics of the repairing and retrofitting materials are presented in Table 1 and Table 2. The main characteristics of the specimens are presented in Figure 1 and Table 3.

Table 1

Mortar characteristics (MAPEI Planitop HDM Maxi)

\begin{tabular}{|c|c|c|c|}
\hline & $\begin{array}{l}\text { Testing } \\
\text { method }\end{array}$ & $\begin{array}{l}\text { Requirements according to } \\
\text { EN1504-3, Class R2 mortars }\end{array}$ & Product performance \\
\hline Compression strength $(\mathrm{MPa})$ : & EN 12190 & $\geq 15$ (after 28 days) & $\begin{array}{c}>3 \text { (after } 1 \text { day) } \\
>15 \text { (after } 7 \text { days) } \\
>25 \text { (after } 28 \text { days) }\end{array}$ \\
\hline Bending strength (MPa): & EN 196/1 & Not required & $\begin{array}{l}>2 \text { (after } 1 \text { day) } \\
>6 \text { (after } 7 \text { days) } \\
>8 \text { (after } 28 \text { days) }\end{array}$ \\
\hline $\begin{array}{l}\text { Elasticity modulus in compression } \\
\qquad(\mathrm{GPa}) \text { : }\end{array}$ & EN 13412 & $\geq 20$ (after 28 days) & 11 (after 28 days) \\
\hline $\begin{array}{c}\text { Concrete bond (MC } 0.40) \text { - water } \\
\text { to cement ratio of } 0.40 \text { according } \\
\text { to EN } 1766(\mathrm{MPa}) \text { : }\end{array}$ & EN 1542 & $\geq 0.8$ (after 28 days) & $>2$ (after 28 days) \\
\hline
\end{tabular}

Table 2

Fiber grids characteristics

\begin{tabular}{|c|c|}
\hline \multicolumn{2}{|c|}{ Glass fiber grids (Mapegrid G 120) } \\
\hline Fiber type: & Alkali resistant fiberglass \\
\hline Weight (g/m2): & 125 \\
\hline Grid spacing (mm): & $12.7 \times 12.7$ \\
\hline Maximum load per unit length (kN/m): & $>25$ \\
\hline Ultimate tensile strain (\%): & $<3$ \\
\hline Carbon fiber grids (Mapegrid C 170) \\
\hline Weight (g/m2): & High-strength carbon fiber \\
\hline Grid spacing (mm): & 170 \\
\hline Ultimate tensile strain (\%): & $5 \times 5$ \\
\hline
\end{tabular}


WMET1

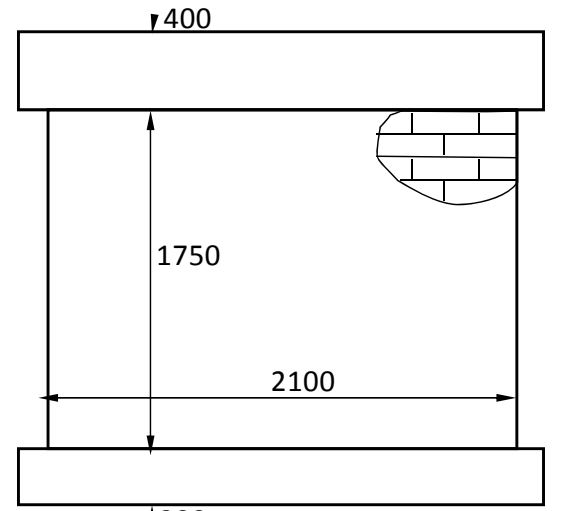

1300

WMET3

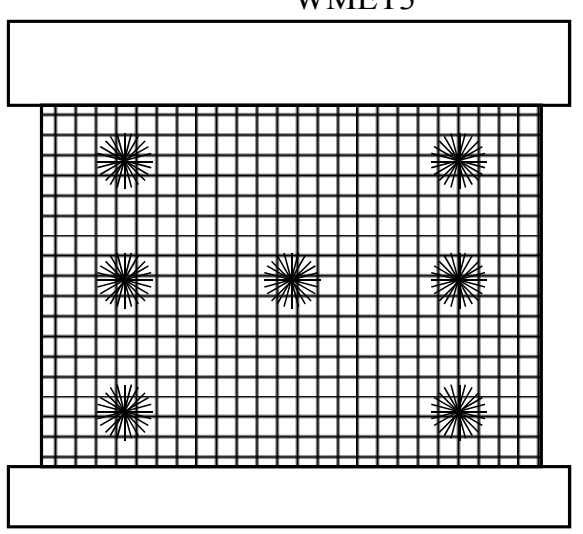

WMET6

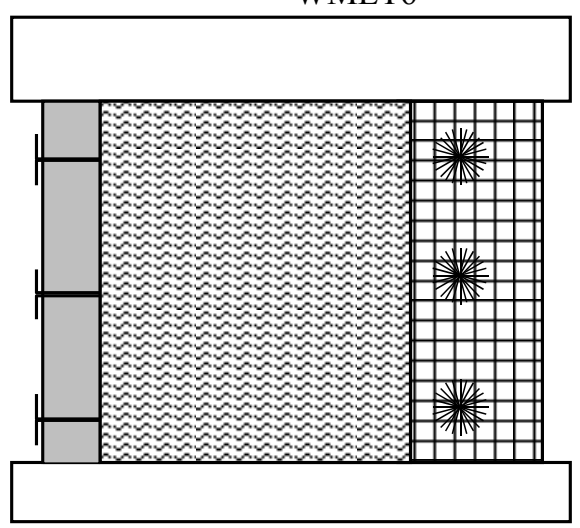

WMET8

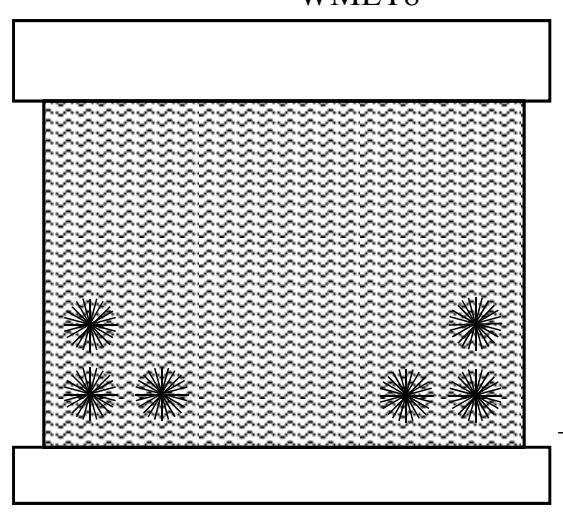

WMET2
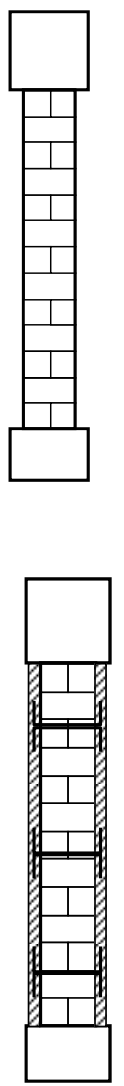

WMET4

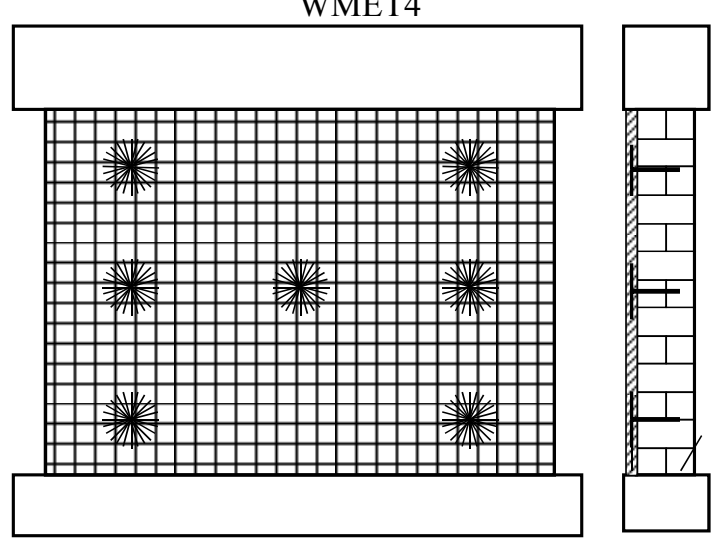

WMET7
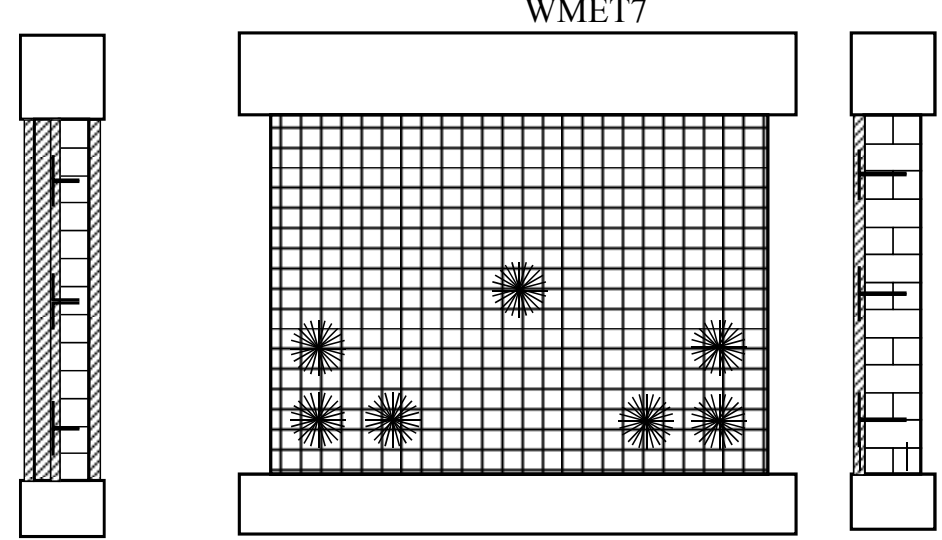

Anchorage on opposite face
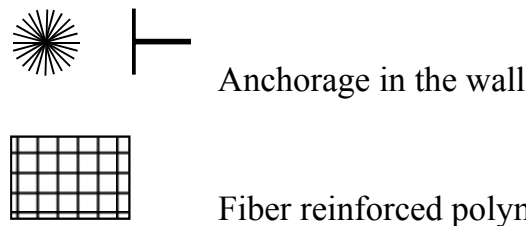

Fiber reinforced polymers grids

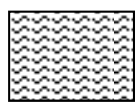

Fiber reinforced mortar

Fig. 1 - Simplified representation of the specimens 
Specimen characteristics

\begin{tabular}{|c|c|c|c|c|c|c|c|}
\hline & WMET1 & WMET2 & WMET3 & WMET4 & WMET6 & WMET7 & WMET8 \\
\hline Repaired: & NO & YES & NO & NO & NO & NO & NO \\
\hline Retrofitted: & NO & YES & YES & YES & YES & YES & YES \\
\hline FRM & NO & YES & YES & YES & YES & YES & YES \\
\hline CFRP : & & NO & NO & 2 layers & 2 layer & 2 layer & NO \\
\hline GFRP & & 2 layers & 2 layers & NO & NO & NO & NO \\
\hline Applied on: & & Both faces & Both faces & One face & One face & One face & Both faces \\
\hline
\end{tabular}

Displacement controlled quasi-static cyclic loading was performed. The specimens were subjected to a cyclic, statically applied, lateral force under a constant axial load. The lateral force was applied using two horizontal 100t hydraulic jacks. The lateral loading protocol included one cycle at $\pm 0,25 \%$ lateral drift and two cycles for each peak at $\pm 0.05 \%, \pm 0.1 \%, \pm 0.2 \%, \pm 0,4 \%$, $\pm 0,6 \%, \pm 1,0 \%, \pm 1,5 \%$. After $1,5 \%$, the lateral displacement was increased up to failure ("pushover" loading). The lateral load protocol is presented in Figure 2.

The axial load was applied using one 200t vertical jack. A mean axial stress of 1,2MPa was applied at the beginning of the test and maintained constant up to failure. The corresponding axial force was $750 \mathrm{kN}$ for all specimens except for WMET6 where the axial force was $420 \mathrm{kN}$ to take into account for the smaller width of the web. Tests were stopped when the loss in the axial force carrying capacity occurred. The reinforced concrete boundary ties at both ends of the specimen were fixed against rotation. A simplified representation of the measurement system is presented in Figure 3.

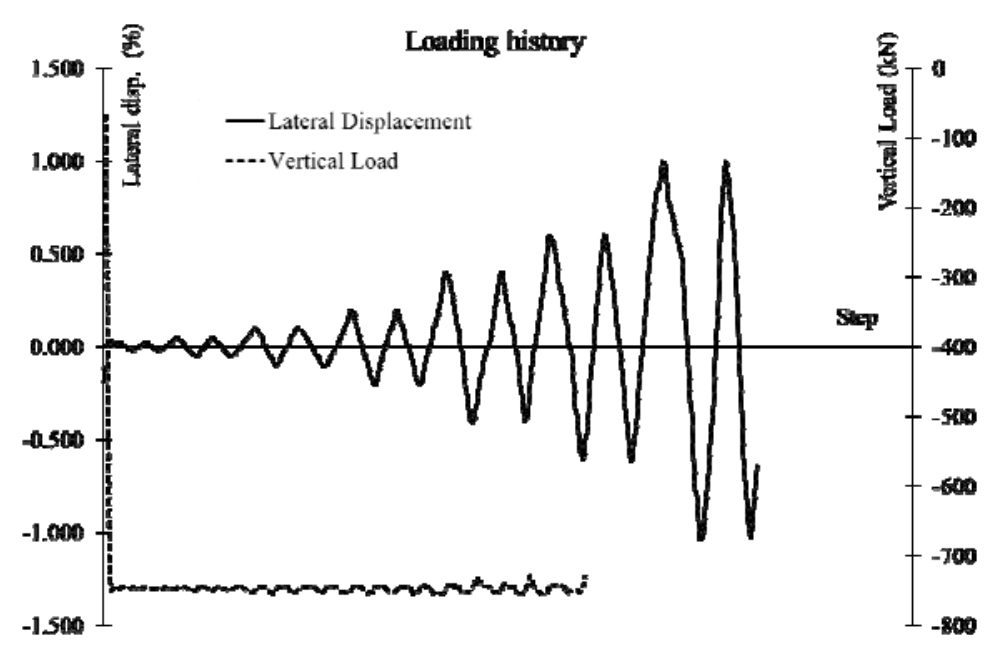

Fig. 2 - Loading protocol

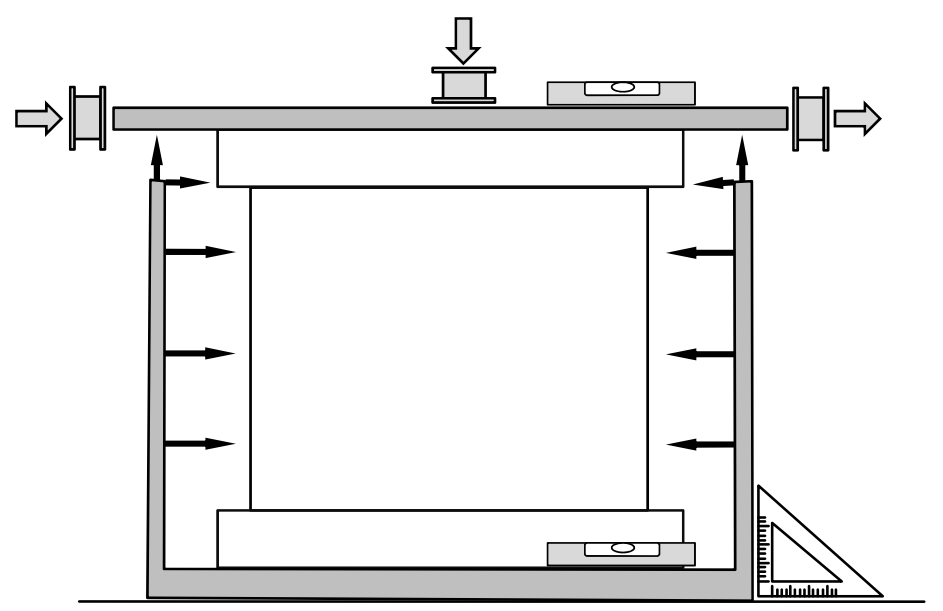

Fig. 3 - Loading and measurement 


\section{Failure Patterns}

Simplified representations of the failure modes are given in Figure 4.

In case of WMET 1 a diagonal failure was noticed. At the first deformation cycle at $0,4 \%$ lateral drift, the diagonal cracking process initiated. In the subsequent cycle, cracks developed along the other diagonal. Considering the damage state of the specimens, after two loading cycles at $\pm 4 \%$ lateral drift, loading was stopped.

After the loading test, WMET1 was repaired and retrofitted. This retrofitted specimen was called WMET2. FRM jacketing with one layer of GFRP grids on each side of the wall was applied. The first diagonal cracks at the surface of the mortar jacket were noticed at the second loading cycle at $-0,4 \%$ drift. These cracks had different orientation in comparison with WMET1 starting from the midpoint of the upper side to the right corner at the bottom side. In the following cycle a symmetrical crack starting from the left-bottom corner appeared. Subsequently, at the following cycles at $0,6 \%$ and $1,0 \%$ inclined cracks along the main diagonals appeared. At 1,36\% drift slip was noticed in the horizontal joint between the masonry wall and the bottom concrete boundary tie and the loading test was stopped.

Specimen WMET3 was obtained by retrofitting an undamaged masonry wall using the same techniques as for specimen WMET2. At 1\% lateral drift slip at the horizontal joint between the masonry wall and the bottom concrete tie was noticed. The corresponding lateral force was approximately $500 \mathrm{kN}$ indicating an equivalent friction coefficient of 0,7 . No cracks at the surface of the mortar jacket and no damage to the bottom corners of the masonry wall were noticed.

Specimen WMET4 was obtained by retrofitting an un-damaged masonry wall by applying a FRM jacket embedding 2 layers of CFRP grids on one face of the wall. Loading was stopped at $0,8 \%$ lateral drift, during the first loading cycle to $+1 \%$. At $+0,6 \%$ drift a sudden decrease of the lateral strength followed by a severe increase of the vertical deformation was recorded. No cracks could be seen at the surface of the FRM jacket. On the opposite side, at the second loading cycle at $+0,4 \%$ lateral drift inclined cracks starting from the midpoint of the upper side to both bottom corners were noticed. After two loading cycles at $0,2 \%$ splitting cracks at the bottom corners of the masonry walls appeared.

In case of WMET6, a vertical crack separating the web from the end zone of the wall was noticed at $0,3 . .0,4 \%$ lateral drift. Subsequently, a sudden decrease of the lateral force of approximately $30 \%$ from the maximum one was recorded. Further loading up to $0,6 \%$ drift increased the wall damage until the loss of the vertical load carrying capacity. Retrofitting at one end on the cross-section by FRP wrapping prevented the deterioration of this end under the applied compression stress.

In case of WMET 7, during the first loading cycle to $-1 \%$, at $0,6 \%$ lateral drift, the first major fracture occurred. A lateral force decay of app. 30\% was recorded. After this event, loading to $1 \%$ lateral drift was continued. As failure to vertical load was observed, after the first loading cycle to $1 \%$ lateral drift lateral loading was stopped.

WMET8 responded essentially elastically up to $0,4-0,6 \%$ lateral drift. At the peak of the first loading cycle to $-0,6 \%$ drift first diagonal cracks were noticed. At the peak of the second cycle to $-0,6 \%$ drift, failure of the wall by a diagonal crack developed from the midpoint of the upper side to the bottom-left corner was noticed. 
Maximum recorded values for loads and displacements in tests for each specimen are given in Table 4.

WMET1 - Diagonal cracking: -0,41\% lateral drift

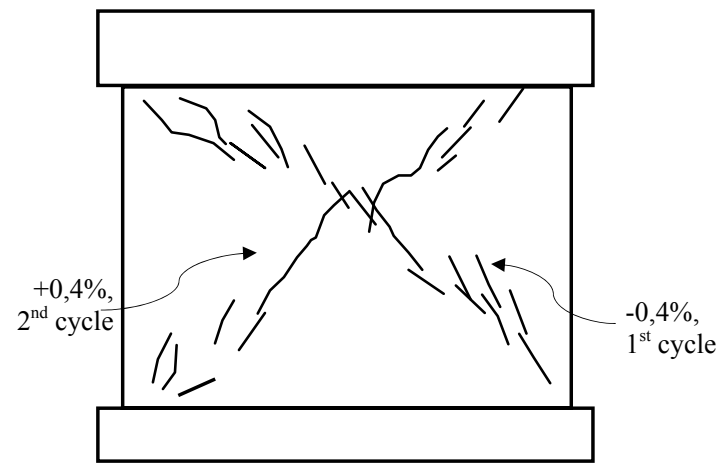

WMET3 - Sliding in the bottom horizontal joint, no major masonry damage (loading stopped at $1,03 \%$ lateral drift)

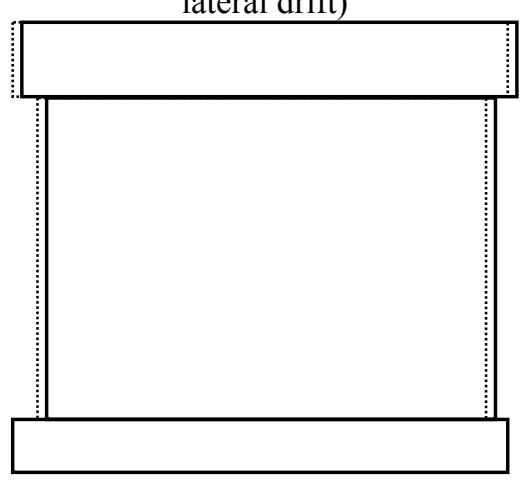

WMET6 - Failure by diagonal cracking followed by the separation of the end zone at $0,61 \%$ lateral drift

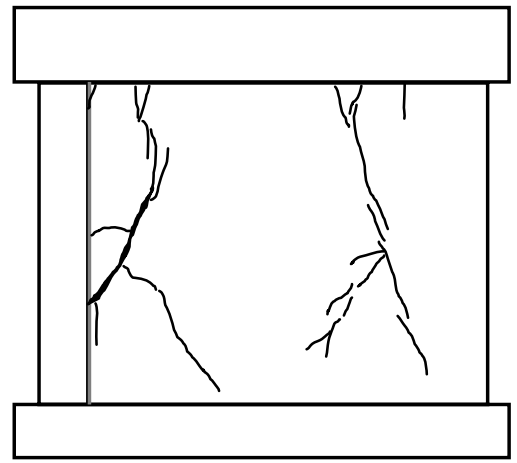

WMET8 - Failure by diagonal cracking at $0,62 \%$ lateral drift

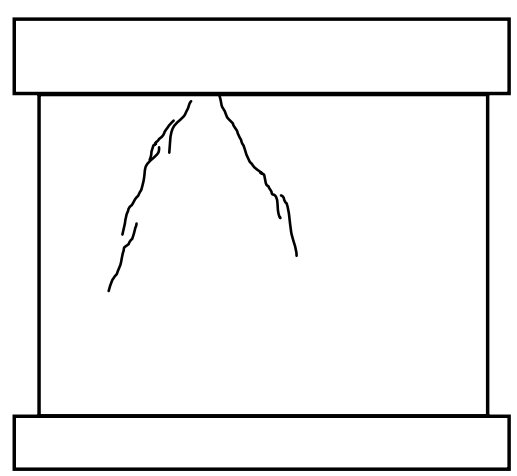

WMET2 -Diagonal cracking, splitting of the web at the bottom corners, sliding in the bottom horizontal joint:

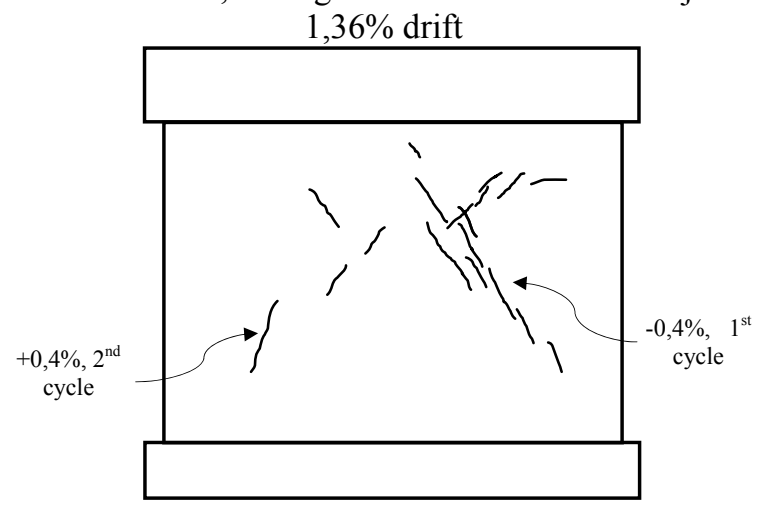

WMET4 - Failure by diagonal cracking followed by web splitting at the bottom corners $(0,8 \%$ lateral drift)
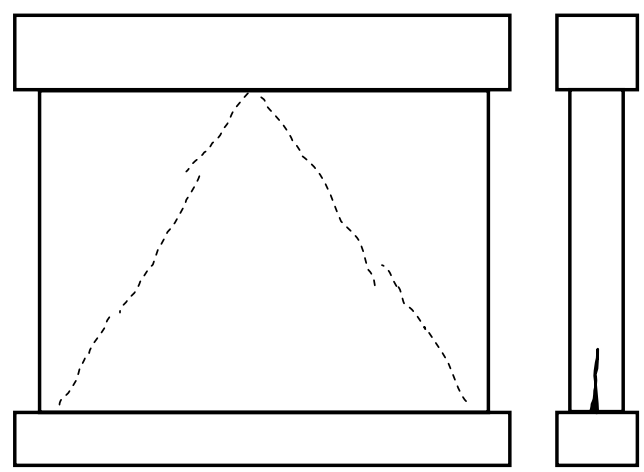

WMET7 - Failure by diagonal cracking with no significant damage at the bottom corners at $1,02 \%$ lateral drift

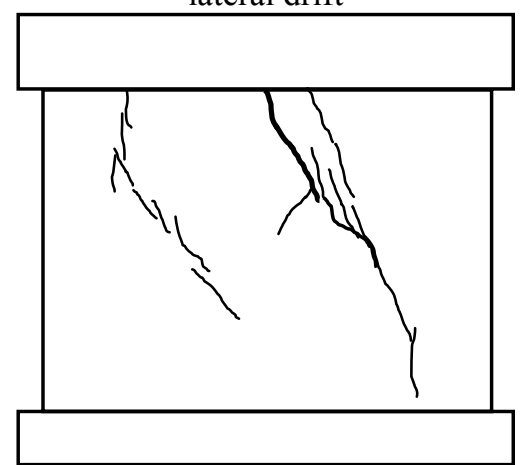

Fig. 4 - Failure patters and peak lateral displacements 
Peak forces and displacements

\begin{tabular}{|l|c|c|c|c|c|c|c|}
\hline & WMET1 & WMET2 & WMET3 & WMET4 & WMET6 & WMET7 & WMET8 \\
\hline $\begin{array}{l}\text { Peak lateral } \\
\text { displacement }\end{array}$ & $-0.41 \%$ & $1.36 \%$ & $-1.03 \%$ & $0.8 \%$ & $0.61 \%$ & $1.02 \%$ & $0.62 \%$ \\
\hline $\begin{array}{l}\text { Peak lateral force } \\
\text { (positive) }\end{array}$ & 452 & 534 & 539 & 494 & 258 & 409 & 467 \\
\hline $\begin{array}{l}\text { Peak lateral force } \\
\text { (negative) }\end{array}$ & -415 & -555 & -470 & -478 & -206 & -419 & -491 \\
\hline
\end{tabular}

\section{Conclusions}

The failure mode of the retrofitting masonry walls strongly depends on the retrofitting details. All the specimens were retrofitted using the same general jacketing solution but different details were considered. The failure modes were different.

Jacketing both sides of the masonry wall with a moderate amount of glass or carbon fibers resulted in the best behavior.

The failure mechanism of the jacketed masonry walls strongly depends on the jacketing details. Two faces jacketing with a moderate amount of fibers gives the best results. In case of WMET3, sliding in the horizontal joint at the interface with the RC support element was observed. After the occurrence of sliding, the lateral force was constant and the damage state was stable.

FRP jacketing improved the capacity of diagonal compression strut. The increased diagonal compression force led to the failure of the web at the corners under compression. The diagonal strut had the smallest width at the corners so a high diagonal compression stress developed in this area. While failure of the web by diagonal cracks is prevented by FRP, a splitting crack appeared in the midplane of the wall (Figure 5). This crack was parallel to the compression stress in the diagonal strut. In case of specimens WMET2 and WMET4 this failure mechanism was observed. If the FRP jacket is bended around the corners of the wall and anchored on the opposite face (Figure 6,a), the efficiency of the jacketing is highly improved. This is not possible in many practical situations, as the wall is usually confined by other walls or columns. To obtain a proper anchorage of the fiber grids to the masonry walls steel wire anchors (Figure 6,b) were also used. A lower efficiency of this anchorage system was observed during the tests.
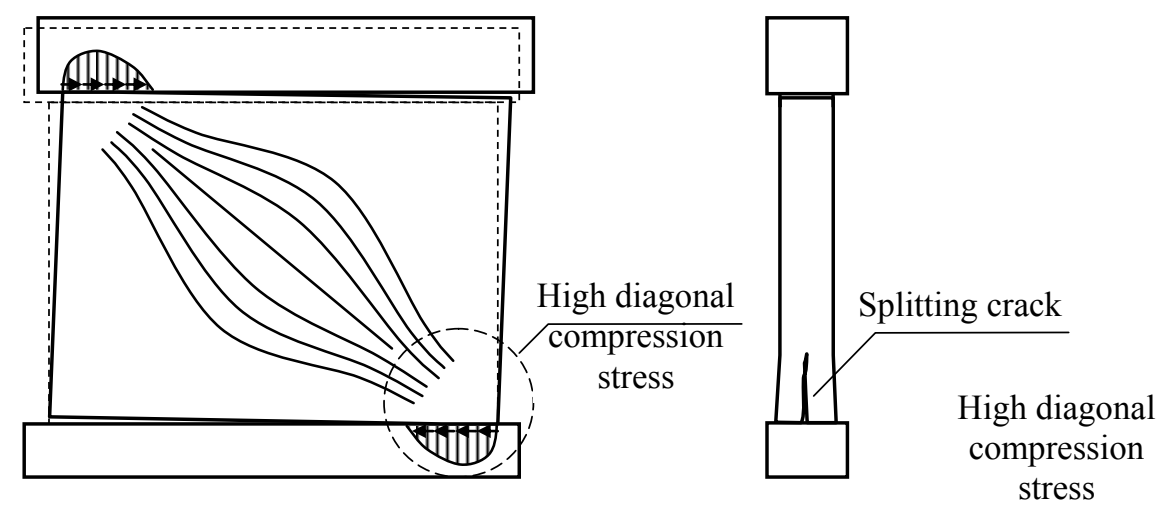

Fig. 5 - Failure by splitting of the web at the bottom corner 


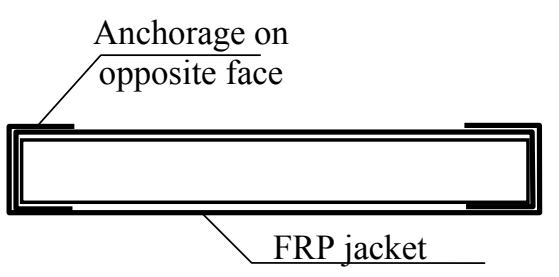

a) Standalone wall

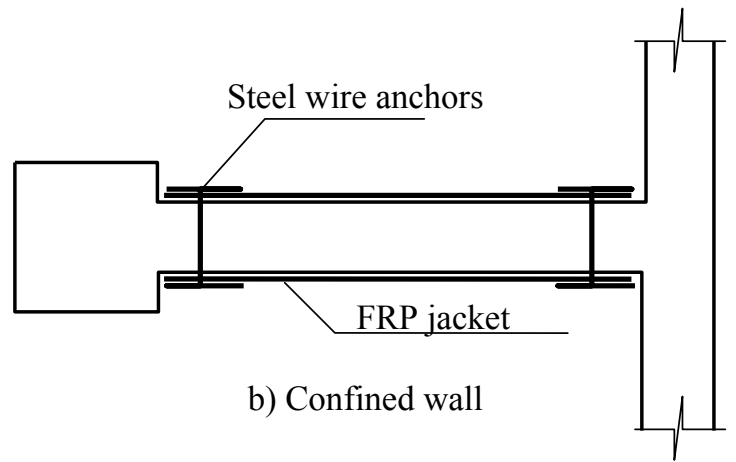

b) Confined wall

Fig. 6 - Anchorage options for FRP jackets

A comparison between the tested specimens and similar un-retrofitted masonry walls, previously tested in the laboratory [6,7], shows that the displacement capacity and lateral strength are not necessarily improved in a satisfactory manner by FRP jacketing. For un-retrofitted masonry walls ultimate lateral drift angles of app. 0,6\% were obtained. For retrofitted specimens values between $0,8 \%$ and $1,4 \%$ were observed. In case of specimen WMET4, retrofitted by FRP jacketing on one face with no proper confinement of the corners in transversal direction, an insignificant increase of the lateral displacement capacity was observed. The strength improvement of app. $20 \%$ cannot compensate the insignificant increase of the displacement capacity.

The results of this testing program will be further analyzed and reported.

\section{Acknowledgements}

The experimental research program was partially supported by the Ministry of Education through the National Center for Programs Management (grant 31034/2007).

MAPEI Romania provided the construction materials and completed the retrofitting works. This support is gratefully acknowledged.

\section{References}

[1] ElGawady M., Lestuzzi P., Badoux M. (2004). A review of conventional seismic retrofitting techniques for URM. In Proceedings of $13^{\text {th }}$ international brick and block masonry conference, 4-7 July 2004 (Paper No. 89). Amsterdam, Netherland. Eindhoven University of Technology.

[2] ElGawady M., Lestuzzi P., Badoux M. (2004). A review of retrofitting of unreinforced masonry walls using composites. In Proceedings of $4^{\text {th }}$ international conference on advanced composite materials in bridges and structures, 20-23 July 2004. Calgary, Canada. Canadian Society of Civil Engineering.

[3] Triantafillou T.C. (1998). Strengthening of masonry structures using epoxybonded FRP laminates. J Comp Constr ASCE (May 1998), 96-104.

[4] Ilki A, Ispir M., As F., Demir C., Kumbasar N. (2008). FRP Retrofit of Walls Constructed with Historical Bricks. In Torres Marques et al. (Eds), CCC2008 - Challenges for Civil Construction, Porto, FEUP,

[5] Aldea C.-M., Mobasher B., and Jain N. (2007). Cement-Based Matrix-Grid System for Masonry Rehabilitation. In ACI Symposium "Thin Fiber and Textile Reinforced Cementitious Systems", 2007, (SP 244CD:9). American Concrete Institute.

[6] Popa V., Cotofana D., Lozincă E., Cheşcă B., Văcăreanu R., Seki M. (2010) Experimental Study on Confined Masonry Squat Walls. In proceedings of the $14^{\text {th }}$ European Conference on Earthquake Engineering, 31 August 03 September. Ohrid, Macedonia, Macedonian Association of Earthquake Engineering

[7] Seki M., Vacareanu R., Saito T., Coțofană D., Lozincă E., Popa V.,Cheșcă A. B. (2008). Cyclic shear tests on plain and FRP retrofitted masonry. In Proceedings of the $14^{\text {th }}$ Word Conference on Earthquake Engineering, $12-$ 17 October 2008. Beijing, China, International Association of Earthquake Engineering 